\title{
Professional Medical Writing: A Tool for High Quality Publications
}

\author{
Medical Writing Profissional: Uma Ferramenta para Publicações de Alta \\ Qualidade
}

João BISSAU $\triangle^{1}$, Pedro BORREGO ${ }^{1,2,3}$

Acta Med Port 2015 Sep-Oct;28(5):545-547

Keywords: Authorship; Interprofessional Relations; Periodicals as Topic.

Palavras-chave: Autoria; Publicações Periódicas; Relações Interprofissionais.

A central aspect of medicine is to disseminate new medical knowledge gained through clinical experience and research, as this is essential for the continuous development of clinical practice. This dissemination may be done, for example, by publishing articles (i.e. case reports, original articles, and literature reviews) in peer reviewed journals. However, to do this may prove challenging for some clinical researchers, as it requires a very specific set of skills and it is very time consuming. In fact, it is known that between 30 and $40 \%$ of clinical research in oncology is never published, and the main reason presented for this is lack of time. ${ }^{1}$ Furthermore, it is also known that numerous papers are rejected by biomedical journals due to poor content organization and writing style. For these reasons, the importance of medical writing skills has been underlined in a recent Portuguese report about successful publishing in biomedical journals, ${ }^{2}$ and many researchers seek professional medical writers (PMWs) for the preparation and submission of articles to biomedical journals. Indeed, according to CenterWatch analysis, the medical writing market in the US more than doubled between 2008 and 2009 , increasing from an estimated $\$ 345$ million to $\$ 694$ million. ${ }^{3}$

\section{Professional medical writing services}

Medical writing is the communication of scientific and clinical information in a clear, unbiased, accurate, and effective fashion, addressing the communicational needs of target audiences (e.g. clinicians, patients, regulators, scientists). Professional medical writing services include the preparation of different types of documents: regulatory (e.g. clinical study protocols, and reports); publications (e.g. articles); medical education (e.g. posters, slide sets for medical meetings); medico-marketing (e.g. promotional product literature). For the purpose of this article, we will only focus on the role of PMWs in publications.

\section{Professional medical writers: background, knowledge,} skills, and abilities

PMWs usually have a background in biomedical sciences (e.g. physicians, nurses, and pharmacists). Often they have advanced degrees (e.g. PhDs) and, therefore, a robust understanding of science, clinical research, and medical communications. ${ }^{4}$ In addition, PMWs may undertake professional development programmes provided by the European Medical Writing Association (EMWA), or by the American Medical Writing Association (AMWA), and are awarded certificates in different subject streams (e.g. drug development, language writing, medical communications, medical sciences, and professional techniques). Due to the increasing complexity of medical writing, it has recently been announced that, starting from 2015, the Medical University Innsbruck will start offering MSc programmes in medical writing.

The knowledge, skills and abilities of PMWs have been clearly presented in several publications by EMWA ${ }^{5}$ and AMWA. ${ }^{6,7}$ Briefly, PMWs are experts in scientific writing and editing, and have a robust knowledge about biomedical sciences (e.g. medicine, pharmacology, and pharmacokinetics), statistics, publication guidelines, and reporting guidelines.

Furthermore, PMWs are familiar with methods for assessing the risk of bias of research. ${ }^{6}$ Therefore, PMWs are able to robustly report clinical research, describing the measures taken to minimize bias, and potential methodological limitations. This improves article acceptance by peer reviewed journals, and contributes for published articles to be scrutinized fairly by, for example, Cochrane reviewers.

PMWs are seldom experts about the concerned therapeutic area. ${ }^{8}$ This may be an advantage, because they will be able to easily identify, and fill in gaps, in the flow of ideas that clinical researches may leave behind, for not realizing that many readers will not be familiar with

\footnotetext{
1. Scientific ToolBox Consulting. Lisbon. Portugal.

2. Instituto de Investigação do Medicamento (iMed.ULisboa). Faculdade de Farmácia. Universidade de Lisboa. Lisboa. Portugal.

3. Centro de Investigação Interdisciplinar Egas Moniz. Instituto Superior de Ciências da Saúde Egas Moniz. Monte de Caparica. Portugal.

$\triangle$ Autor correspondente: João Bissau. joao.bissau@sctbx.com

Recebido: 30 de Dezembro de 2014 - Aceite: 26 de Março de 2015 | Copyright @ Ordem dos Médicos 2015
} 
$1 \mathrm{~A}$
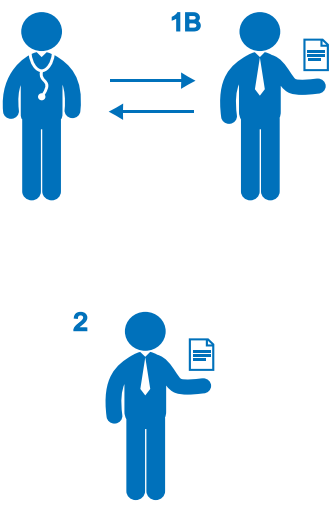

3

4

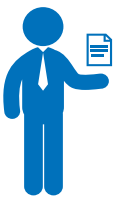

$\uparrow \downarrow$
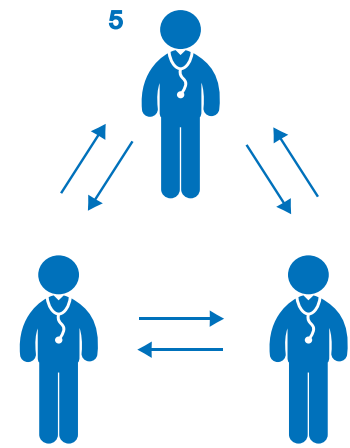

6

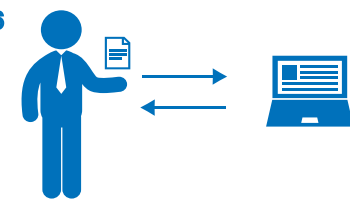

1 A Lead clinical researcher

Provides an overview of the study, and the study documentation to the professional medical writer.

$1 B$

Professional medical writer

Assesses whether it is necessary to: undertake a formal literature review before article preparation; seek a biostatistician and/or a medical illustrator.

Professional medical writer

Advises on the journal to which the article should be submitted to.

3 Lead clinical researcher and professional medical writer Agree on a writing plan establishing the: most important aspects to be reported most important references to be included in the background and discussion sections; deadlines for handing in drafts, draft review, and journal submission.

Professional medical writer

Drafts of the article are prepared and sent to the clinical researchers.

Comments sent by Clinical Researchers are used to prepare updated versions of the article.

5 Clinical researchers

Send comments for the preparation of new versions of the article.

Approve the final version of the article.

Professional medical writer

Submits the final version of the article to the journal.

Submission follow-up.

Figure 1 - Interaction between professional medical writers and clinical researchers

their areas of expertise. Furthermore, PMWs are able to do comprehensive literature searches, to liaise with the clinical researchers and medical consultants, to learn about new therapeutic areas in a timely fashion.

\section{Working with professional medical writers}

Team work between clinical researchers and PMWs is a strategy for preparing high quality articles (see Fig. 1). ${ }^{9}$ Firstly, clinical researchers provide PMWs an overview of the study (e.g. background, research questions, main methods, and results), and study documentation (e.g. protocol, reports, abstracts, posters, and slide sets). This allows PMWs to assess whether it is necessary to undertake a formal literature review before article preparation, and/or 
to seek support of other professionals (e.g. biostatistician, medical illustrator). Furthermore, this also allows PMWs to plan the best way to convey the message of the study for the desired target audience. ${ }^{5}$

Secondly, PMWs advise on the right journals for a particular article. This is likely to speed up publication times, because it will avoid the submission of articles to journals whose editors are likely to reject them, for feeling that these are out of the scope of the journal, and not likely to be highly cited by their target audience. Quick publication times are important for decreasing the probability that similar studies are published first, diminishing the novelty of the subject matter.

Thirdly, clinical researchers and PMWs agree on a writing plan. This establishes the most important aspects to be reported, the most relevant bibliographic references to be included in the background and discussion sections, and sensible deadlines for handing in drafts, draft review, and journal submission. This is pivotal for assuring that PMWs are aware of the clinical researchers' expectations, and that these will be met.

Then, PMWs prepare a draft of the article based on the clinical information provided by the researchers, taking into account, for example, the requirements of the journal that the article is going to be submitted to, and the most suitable reporting and publishing guidelines. Furthermore, the article is also prepared following the scientific writing style: sentences and paragraphs will be connected following a logical sequence, and medical jargon will be replaced, as far as possible, by a more reader friendly language in order to increase readership.

Next, PMWs send the draft of the article to the clinical researchers for review. These will add comments, suggesting changes for improvement. PMWs may provide a rationale for accepting or rejecting certain comments, but the clinical researchers are usually responsible for the final decisions about these comments. Typically 2 or 3 review rounds are necessary before the final version of the article is ready.

Finally, PMWs submit the final version of article to the selected journal. In some instances, before making a final decision on whether to accept an article or not, journal editors may ask for clarification about certain aspects of the study, and may suggest changes for article improvement. PMWs advise clinical researchers on the best course of action, prepare a new version of the article, and resubmit it. If the article fails to be accepted by that journal, PMWs advise on an alternative journal to which the article should be submitted to.

\section{Professional medical writers and authorship}

Generally, PMWs do not contribute substantially to the conception or design of clinical research, not meeting the ${ }^{I C J M E}{ }^{10}$ criteria for authorship. Therefore, they are not to be listed as authors, but their contribution should be presented in the acknowledgments section. ${ }^{10}$ This promotes transparency, helping readers, reviewers, and journal editors to understand how the manuscript was prepared. ${ }^{5}$

\section{CONCLUSION}

PMWs have a background in biomedical sciences and are likely to have advanced degrees. They combine their knowledge in science, clinical research, and medical communications, to prepare articles using the most suitable language for the target audience. Finally, they have training in publication and reporting guidelines, and are able to suggest the right journal for a particular article.

\section{ACKNOWLEDGEMENT}

Scientific ToolBox Consulting created Figure 1 using source material made by Freepik from http://www.flaticon. com.

\section{CONFLICTS OF INTEREST}

The authors declare that they are employed by Scientific ToolBox Consulting.

\section{FUNDING SOURCES}

None stated.

\section{REFERENCES}

1. Camacho LH, Bacik J, Cheung A, Spriggs DR. Presentation and subsequent publication rates of phase I oncology clinical trials. Cancer. 2005;104:1497-504

2. Marinho RT, Donato H, Fernandez-Llimos F, Massano J, Silva JM, Almeida $\mathrm{M}$, et al. Think Tank: Relatório estratégico sobre publicação científica biomédica em Portugal. Acta Med Port. 2014;27:1-3.

3. The Centerwatch Monthly. Demmand for medical writing continues to rise. 2008. [Accessed 2014 Dec 1]; Available from: http://www. centerwatch.com/advertise/sampleMonthly.pdf.

4. Das N, Das S. Hiring a professional medical writer: is it equivalent to ghostwriting? Biochem Med. 2014;24:19-24

5. Jacobs A, Wager E. European Medical Writers Association (EMWA) guidelines on the role of medical writers in developing peer-reviewed publications. Curr Med Res Opin. 2005;22:317-21.

6. Clemow DB. Pharmaceutical medical writing competency model. AMWA J. 2011;26:62-70.

7. Hamilton CW, Royer G. AMWA position statement on the contributions of medical writers to scientific publications. AMWA J. 2003;18:13-6.

8. Marchington JM, Burd GP. Author attitudes to professional medical writing support. Curr Med Res Opin. 2014;30:2103-8.

9. Hindle A, Tobin SC, Robens J, McGowan D. Working with authors to develop high-quality, ethical clinical manuscripts: Guidance for the professional medical writer. Med Writ. 2014;23:228-35.

10. International Committee of Medical Journal Editors (ICMJE). Recommendations for the Conduct, Reporting, Editing, and Publication of Scholarly Work in Medical Journals. 2014. [Accessed 2014 Dec 1]; Available from: http://www.icmje.org/icmje-recommendations.pdf. 


\section{Professional Medical Writing: A Tool for High Quality Publications}

Acta Med Port 2015:28:545-547

Publicado pela Acta Médica Portuguesa, a Revista Científica da Ordem dos Médicos

Av. Almirante Gago Coutinho, 151

1749-084 Lisboa, Portugal.

Tel: +351218428215

E-mail: submissao@actamedicaportuguesa.com

www.actamedicaportuguesa.com

ISSN:0870-399X | e-ISSN: 1646-0758

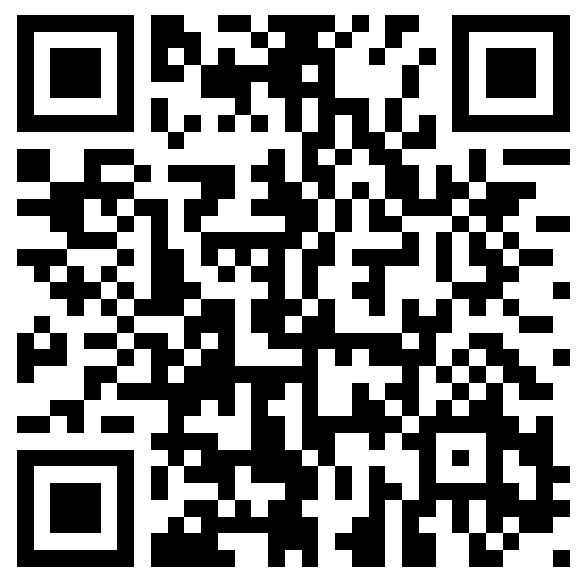

